\title{
Review:
}

\section{Current status and future prospects of mesenchymal stem cell therapy for liver fibrosis*}

\author{
Yang GUO ${ }^{\dagger}$, Bo CHEN, Li-jun CHEN, Chun-feng ZHANG, Charlie XIANG ${ }^{\dagger *}$ \\ (State Key Laboratory for Diagnosis and Treatment of Infectious Diseases, and Collaborative Innovation Center for Diagnosis and \\ Treatment of Infectious Diseases, the First Affiliated Hospital, School of Medicine, Zhejiang University, Hangzhou 310003, China) \\ †E-mail: mavis199005@zju.edu.cn; cxiang@zju.edu.cn \\ Received Mar. 3, 2016; Revision accepted May 14, 2016; Crosschecked Oct. 10, 2016
}

\begin{abstract}
Liver fibrosis is the end-stage of many chronic liver diseases and is a significant health threat. The only effective therapy is liver transplantation, which still has many problems, including the lack of donor sources, immunological rejection, and high surgery costs, among others. However, the use of cell therapy is becoming more prevalent, and mesenchymal stem cells (MSCs) seem to be a promising cell type for the treatment of liver fibrosis. MSCs have multiple differentiation abilities, allowing them to migrate directly into injured tissue and differentiate into hepatocyte-like cells. Additionally, MSCs can release various growth factors and cytokines to increase hepatocyte regeneration, regress liver fibrosis, and regulate inflammation and immune responses. In this review, we summarize the current uses of MSC therapies for liver fibrosis and suggest potential future applications.
\end{abstract}

Key words: Liver fibrosis, Cell therapy, Mesenchymal stem cells http://dx.doi.org/10.1631/jzus.B1600101

CLC number: R575

\section{Introduction}

Liver fibrosis is a pathologic process that occurs between liver injury and liver cirrhosis. After liver injury, several processes occur including cell apoptosis, inflammation, and scarring, resulting in the deposition of the extracellular matrix (ECM). In the early stages, the ECM deposition can be hydrolyzed by proteolytic enzymes such as matrix metalloproteinases (MMPs). However, continuous damage will ultimately lead to excessive matrix deposition and the alteration of the normal liver structure (Lichtinghagen et al., 2001).

Liver fibrosis can be triggered by viruses, alcohol abuse, drug abuse, and auto-immunity, among

\footnotetext{
${ }^{\ddagger}$ Corresponding author

* Project supported by the National High-Tech R \& D Program (863) of China (No. 2015AA020306)

(iD) ORCID: Yang GUO, http://orcid.org/0000-0002-2125-0637

(C) Zhejiang University and Springer-Verlag Berlin Heidelberg 2016
}

other conditions. When liver fibrosis is not well controlled, it can develop into liver cirrhosis, which is the end-stage of liver disease. Currently, liver transplantation is the main effective therapy for liver cirrhosis, but this treatment method is associated with many problems, such as immunological rejection, donor shortages, surgical complications, and high costs (Eom et al., 2015). Therefore, finding new therapeutic strategies for liver fibrosis is essential.

In addition to liver transplantation, cell therapy is a common treatment method for liver disease. For instance, hepatocyte transplantation can be used to restore liver function because of the regeneration abilities of these cells. However, the utility of this treatment is limited because hepatocytes easily lose their viability and function when they are cultured in vitro or when they are preserved cryogenically (Eom et al., 2015). Thus, other types of cells have been explored in an effort to find an ideal treatment for liver diseases. Such research has shown that stem cell transplantation is an effective therapy for liver fibrosis 
(Kakinuma et al., 2009; Kharaziha et al., 2009). Among the different types of stem cells, mesenchymal stem cells (MSCs) in particular have obvious advantages in regenerative repair because of their high potential for multipotent differentiation, capacity for self-renewal, and low immunogenicity (Jang et al., 2014). MSCs are fibroblast-like and plate-adhering cells, which have the ability to self-renew and to differentiate into adult cells from different germ layers, such as neurocytes from ectoderm, osteoblasts and myocytes from mesoderm, and hepatocyte-like cells from endoderm (Chan et al., 2014). Recently, MSCs have been isolated from a variety of tissues including umbilical cord blood, adipose tissue, liver, lung, dermis, amniotic membrane, and menstrual blood (Erices et al., 2000; Campagnoli et al., 2001; Jiang et al., 2002; de Ugarte et al., 2003; Mou et al., 2013). Additionally, MSCs can secrete a series of cytokines and signaling molecules, such as hepatocyte growth factor (HGF), interleukin 6 (IL-6), tumor necrosis factor alpha (TNF- $\alpha$ ), epidermal growth factor (EGF), nitric oxide, prostaglandin E2 (PGE2), and indoleamine 2,3-dioxygenase (Ortiz et al., 2007; Kiss et al., 2008; Puglisi et al., 2011), which can regulate inflammatory responses, stimulate hepatocyte proliferation, and maintain hepatocyte function (Lin et al., 2011; Sharma et al., 2014) (Fig. 1).
In general, MSC therapy for liver fibrosis is effective and promising, and many studies have been performed in this field. Thus, in this review, we discuss the current research regarding the mechanisms and uses of MSC therapy for liver fibrosis and the associated limitations, and we suggest some potential future applications of this therapy.

\section{Mechanisms of fibrogenesis in the liver}

The mechanisms of liver fibrosis are complex and involve a variety of cytokines, growth factors, and signaling pathways. Although many studies have been performed, the exact mechanisms of fibrogenesis remain unknown. It has been established that an imbalance between ECM production and degradation is the precipitating cause of liver fibrosis (Ek et al., 2007). However, how the imbalance happens is unclear.

The universally accepted key mechanism involved in ECM accumulation is the activation of transforming growth factor beta (TGF- $\beta$ )/Smad signaling (Wrana, 1999; Berardis et al., 2015), which is mediated by transmembrane serine/threonine kinase receptors including type I and type II. In the injured liver, the microenvironment promotes the activation of Kupffer cells, which in turn exert proinflammatory

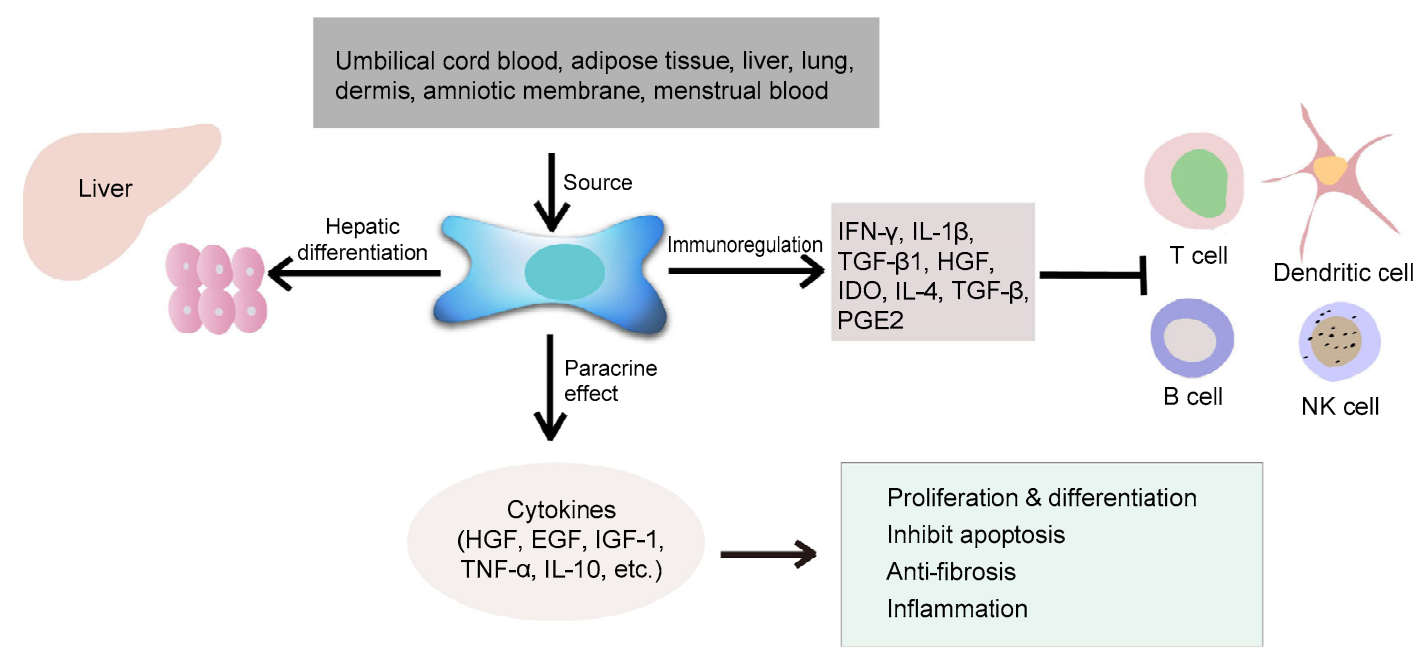

Fig. 1 Sources and function of mesenchymal stem cells (MSCs)

The sources of MSCs are abundant, including umbilical cord blood, adipose tissue, liver, lung, dermis, amniotic membrane, and menstrual blood, among others. MSCs can differentiate into hepatocytes, secrete various cell factors, and participate in immunoregulation. IFN- $\gamma$ : interferon gamma; IL: interleukin; TGF: transforming growth factor; HGF: hepatocyte growth factor; IDO: indoleamine 2,3-dioxygenase; PGE2: prostaglandin E2; EGF: epidermal growth factor; IGF: insulin-like growth factor; TNF: tumor necrosis factor; NK cells: natural killer cells 
cytokines such as TNF- $\alpha$, TGF- $\alpha$, TGF- $\beta$, and plateletderived growth factor (PDGF), among others. The increased TGF- $\beta$, combined with the type II receptor, activates the type I receptor and forms a complex. Then, the complex phosphorylates, for the downstream signal transduction molecules $\operatorname{Smad} 2 / 3$, are translocated into the nucleus where they regulate transcriptional responses such as collagens.

The activated hepatic stellate cells (HSCs), which can transform into myofibroblast-like cells, also play a critical role in the production of ECM (Berardis et al., 2015). When stimulated by lipid peroxides, products from injured hepatocytes, or biochemical signals from Kupffer cells, HSCs can become activated and exhibit the following: high expression of alpha smooth muscle actin ( $\alpha$-SMA), tissue inhibitors of metalloproteinases (TIMPs)-1/2, the secretion of collagen-1, and increased proliferation ability (Iredale et al., 1992; Friedman, 1993; Benyon et al., 1996). The activation can occur through autocrine and paracrine signaling pathways; one of the main pathways is the PDGF- $\beta$ signaling pathway. The PDGF- $\beta$ signaling pathway can activate other pathways, such as the Ras-mitogen-activated protein kinase, phosphoinositide 3-kinase-AKT/protein kinase $\mathrm{B}$, and protein kinase $\mathrm{C}$ pathways, resulting in HSC proliferation (Kelly et al., 1991). In addition to HSCs, other cell types, such as circulating fibrocytes, portal fibroblasts, and bone marrow-derived cells, are believed to contribute to ECM deposition (Forbes et al., 2004; Wells et al., 2004).

Recent studies have shown that the development of liver fibrosis is accompanied by the expression of MMPs (Lichtinghagen et al., 2001). MMPs are critical for the regression of fibrogenesis in which they can degrade collagens and are involved in the early stages of tissue remodeling (Milani et al., 1994; Benyon et al., 1996). Moreover, TIMPs, which can be produced by activated HSCs, are believed to induce ECM deposition by slowing the breakdown of collagens (Arthur, 1995; 1997). It is known that the expression of TIMPs is mainly induced by inflammation responses. Inflammation factors like IL- $1 \beta$ and TNF- $\alpha$ can promote TIMP expression. Thus, the expression level and activity of TIMPs can be used as indicators to measure the disease process. In general, the balance between MMPs and TIMPs plays an important role in liver fibrosis (Böker et al., 2000).
Thus, it is clear that the TGF- $\beta /$ Smad signaling pathway plays a critical role in ECM accumulation. When this pathway is activated, the downstream factors notably increase, which induces the expression of fibrosis-related genes including the genes for collagen-1, $\alpha$-SMA (a surface marker of HSCs), and TIMPs. As a result, the number of activated HSCs increases, whereupon the degradation cannot match the production of ECM, resulting in liver fibrosis (Fig. 2).

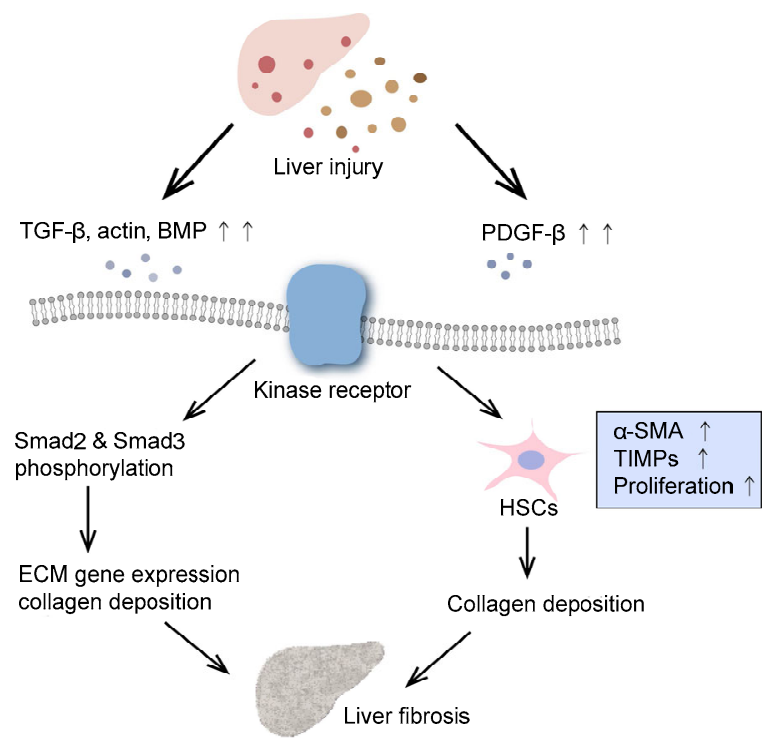

Fig. 2 Signaling pathway of liver fibrogenisis The injured liver produces various cell factors, which can activate transforming growth factor beta (TGF- $\beta$ ) and platelet-derived growth factor beta (PDGF- $\beta$ ), and bone morphogenetic protein (BMP). Through the TGF- $\beta$ signaling pathway, Smad2/3 are phosphorylated and activate downstream molecules such as extracellular matrix (ECM) genes, resulting in the deposition of collagen. Hepatic stellate cells (HSCs) are greatly increased through the PDGF- $\beta$ pathway and they induce the secretion of collagens and tissue inhibitors of metalloproteinases (TIMPs). Thus, the degradation of collagens decreases and the secretion increases, leading to the deposition of collagens, which results in worsened liver disease. $\alpha$-SMA: alpha smooth muscle actin

\section{Differentiation ability of MSCs}

It is known that MSCs have the capacity to differentiate into various progenitor cells from different cell lines, including hepatic progenitor cells. Indeed, a variety of studies (Banas et al., 2007; Ishii et al., 2008; Kakinuma et al., 2009; Puglisi et al., 2011; Hang et al., 2014) have demonstrated the ability of MSCs to 
differentiate into hepatocyte-like cells by examining the expression of specific hepatocyte markers such as albumin, $\alpha$-fetoprotein, and cytokeratin-19, among others.

The ability of MSCs to differentiate into hepatocyte-like cells makes them an ideal alternative method for treating liver fibrosis. Therefore, many studies have examined the mechanisms underlying the differentiation ability of MSCs. Several recent studies (Yoshida et al., 2007; Ishii et al., 2008; Liu et al., 2015) have demonstrated that $\mathrm{Wnt} / \beta$-catenin signaling plays an important role in regulating the hepatic differentiation of human MSCs. Upon Wnt signaling activation, $\beta$-catenin will translocate into the nucleus and coactivate downstream transcription factors to regulate the differentiation of MSCs. Furthermore, mesenchymal-epithelial transition and the reverse, epithelial-mesenchymal transition are critical developmental processes that play fundamental roles in the differentiation of multiple tissues (Hay, 2005). Epigenetic modifications, such as DNA methylation and histone acetylation, have also been shown to participate in the differentiation of MSCs (Snykers et al., 2007).

Additionally, some studies have tried to enhance the efficiency of MSC differentiation, since during regular differentiation, MSCs have low metabolic activity and low expression of functional proteins (Ek et al., 2007). For instance, Mohsin et al. (2011) demonstrated that pretreating MSCs with injured liver tissue enhances their differentiation ability owing to the growth factors and cytokines that are released by the injured tissue, such as HGF, insulin-like growth factor (IGF), EGF, and basic fibroblast growth factor (bFGF), among others (Liu et al., 2015).

While it is clear that MSCs have multidifferentiation abilities including the ability to differentiate into hepatocyte-like cells, it remains unclear whether MSCs can adopt a mature hepatic fate, as no reliable and detailed results of mature hepatocytic gene expression have been reported. Many researchers believe that MSCs can become hepatocytes both morphologically and functionally. For instance, Banas et al. (2007) and Yin et al. (2015) demonstrated that adipose tissue-derived MSCs could be induced into transplantable and mature hepatocytelike cells both in vivo and in vitro. Moreover, these studies showed that differentiated MSCs express hepatocyte-specific markers including albumin and $\alpha$-fetoprotein and share liver functions such as lowdensity lipoprotein uptake, glucose storage, and ammonia detoxification.

In contrast, other researchers have opposed the opinion that MSCs can adopt a mature hepatic fate by claiming that only early specific markers have been detected and noting that little credible data on the detection of mature hepatocyte markers exist. Campard et al. (2008) conducted a study to detect the differentiation ability of umbilical cord matrix stem cells; the results showed that the differentiated umbilical cord matrix stem cells exhibited hepatocytelike morphologies, specific liver markers (e.g., albu$\min , \alpha$-fetoprotein, cytokeratin-19, connexin-32), and some hepatic functions including glucose storage, low-density lipoprotein uptake, and urea production. However, the cells did not express hepatocyte nuclear factor 4 or HepPar1, two specific hepatic makers. In addition, the differentiated MSCs still contained some MSC-specific makers. Collectively, these findings suggested that the differentiated MSCs did not express enough markers of mature hepatocytes, implying that MSCs cannot fully become hepatocytes. Lian et al. (2006) demonstrated that bone marrow (BM) hematopoietic stem cells expressed several hepatic markers but could not be efficiently converted into hepatocyte-like cells, as one of the mature hepatic markers (anti-trypsin) was not detected. Another study (Hengstler et al., 2005), based on drug metabolism, showed that it is unlikely that MSCs fully differentiate into hepatocytes, and it has also noted that the use of different protocols for hepatic differentiation and different detection methods are problematic. Therefore, specific criteria are needed to define hepatocyte-like cells derived from MSCs. It has been suggested that the definition should not only be based on qualitative analyses but also on quantitative analyses including analyses of enzyme activity.

Overall, MSCs have the potential to differentiate into immature hepatocyte-like cells that exhibit some early specific hepatic markers and functions. Regardless, MSCs are an optimal choice for treating liver fibrosis because of their paracrine effects and immunologic regulation in addition to their multidifferentiation potential. 


\section{Paracrine effect of MSCs}

MSCs have the ability to migrate into injured tissues via chemotaxis due to cytokines that are released from the injured organ or tissues (Golzar et al., 2015; Lourenco et al., 2015). Under stimulation of the microenvironment in injured tissue, like some inflammation factors, MSCs can release various growth factors and cytokines, which promote the proliferation of endogenous hepatocytes, reduce hepatocyte apoptosis, enhance liver function, and repress inflammatory responses (Zhou et al., 2009; Lin et al., 2011).

Research shows that MSCs secrete cytokines, such as HGF, EGF, IL-6, and TNF- $\alpha$, which can stimulate hepatocyte proliferation and enhance liver function, as indicated by the high levels of albumin and urea secretion. For instance, Kim et al. (2014) overexpressed HGF by transducing MSCs with an adenovirus vector carrying the HGF gene. Their results showed a decrease in collagen and lower mRNA levels of the fibrogenic cytokines PDGF-bb and TGF- $\beta 1$, suggesting that MSCs that overexpress HGF are effective in the treatment of liver fibrosis. Additionally, MSCs can release other cytokines such as IGF-1, stromal cell-derived factor-1 (SDF-1), and a vascular endothelial growth factor (VEGF), which inhibit cell apoptosis mainly by regulating the SDF-1/CX chemokine receptor-4 (CXCR-4) axis (Lin et al., 2011). IGF-1 is an important factor in body metabolism, and has been demonstrated to be antiapoptotic to hepatocytes and increase the secretion of HGF in the cirrhotic liver (Bonefeld and Møller, 2011). Fiore et al. (2015) used a recombinant adenovirus overexpressing IGF-1 in BM-MSCs to ameliorate liver fibrosis in mice. The application of BMderived AdIGF-I-MSCs resulted in the reduced activation of HSCs, increased IGF-I and HGF expression, reduced fibrogenesis, and increased hepatocyte proliferation.

Moreover, researches (Siller-López et al., 2004; Snykers et al., 2007) have demonstrated that MSCs overexpressing MMPs promote the regression of liver fibrosis. MSCs have the potential to reverse the fibrotic process by inhibiting collagen deposition through high levels of MMPs including MMP-8, MMP-9, and MMP-13. MMPs have been shown to degrade the ECM directly in order to balance the increased TIMPs that are induced by activated HSCs, thus contributing to the regression of fibrogenesis (Lin et al., 2011). In addition, the blockades of MSC-derived IL-10 and TNF- $\alpha$ exhibit minimal inhibitory effects on HSC proliferation and collagen synthesis, demonstrating the anti-fibrogenic effects of IL-10 and TNF- $\alpha$ (Parekkadan et al., 2007).

It is known that cytokines are important factors that participate in inflammation, as they can mediate inflammatory responses and prevent inflammatory effects. TNF- $\alpha$, IL-1, and IL- 6 are familiar proinflammatory factors that play critical roles in activating immunocytes and in regulating tissue metabolism (Liu et al., 2013; Huang et al., 2015). In injured tissue, TNF- $\alpha$ is one of the first factors to be released, which then activates neutrophil granulocytes and lymphocytes and induces the secretion of other inflammation factors. In a lung injury model, MSCs have been shown to express an IL-1 receptor antagonist that blocks the release of TNF- $\alpha$ from activated macrophages, thus preventing tissue damage (Ortiz et al., 2007).

\section{MSC therapy and immunoregulation}

It has been established that MSCs possess remarkable immunosuppressive properties that inhibit the proliferation and function of immune cells from both the adaptive and innate immune systems (Shi et al., 2011). The immunomodulatory effects of MSCs are mediated through both a cell-cell contact and secreted factors such as PGE2, nitric oxide, and TGF- $\beta$. MSCs can also inhibit the proliferation of T lymphocytes through cell contact (Tse et al., 2003; Sotiropoulou et al., 2006) and through soluble cytokines such as HGF, IL-1 $\beta$, TGF- $\beta 1$, interferon gamma (IFN- $\gamma$ ), and indoleamine 2,3-dioxygenase (di Nicola et al., 2002; Meisel et al., 2004; Groh et al., 2005; Krampera et al., 2006), which is indicated by an increase in the number of cells in the G0/G1 phase (Glennie et al., 2005) and by the up-regulated expression of p27 (Krampera et al., 2003). Further, MSCs can inhibit $\mathrm{CD}_{4}^{+} \mathrm{T}$ cells, $\mathrm{CD}_{8}{ }^{+} \mathrm{T}$ cells (Glennie et al., 2005), T-helper lymphocytes (Th1/Th17) (Aggarwal and Pittenger, 2005; Zappia et al., 2005), and cytotoxic T cells (Potian et al., 2003; Rasmusson et al., 2003). The suppression effect of MSCs on T 
cells can indirectly act on B lymphocytes because B cell activation mainly depends on T cells. Additionally, MSCs can directly inhibit the proliferation of B lymphocytes, the production of antibodies, and chemotaxis when co-stimulating with anti-immunoglobulin antibodies, anti-CD40L and IL-4 in humans (Corcione et al., 2006).

MSCs also have suppression effects on cells belonging to the innate immune system, including natural killer (NK) cells, dendritic cells (DCs), monocytes, and macrophages. Studies have shown that MSCs can only partially suppress the proliferation of activated NK cells. Some cell factors such as TGF- $\beta 1$ and PGE2 are believed to participate in the suppression of NK cell proliferation (Rasmusson et al., 2003; Krampera et al., 2006). In addition, MSCs can affect the production of DCs by inhibiting the differentiation of monocytes, as MSCs can block the maturation signals and co-stimulatory molecules (Zhang et al., 2004; Jiang et al., 2005; Nauta et al., 2006). On the other hand, MSCs reduce the proinflammatory ability of DCs by decreasing the secretion of TNF- $\alpha$, IFN- $\gamma$, and IL-12 and increasing IL-10 secretion (Zhang et al., 2004; Jiang et al., 2005).

In summary, the ability of MSCs to regulate immune responses is an important advantage for cell therapy and allogeneic transplantation. It is known that MSCs have low immunogenicity because they lack human leukocyte antigen class II and co-stimulatory molecules such as CD80, CD86, and CD40 in the cytomembrane (Reinders et al., 2013). In addition, the sources of MSCs are various and abundant. MSCs also have direct migration abilities and a high differentiation capacity. Considering all of these characteristics, MSCs are the ideal transplant donors in regeneration diseases.

\section{MSC therapy and CRISPR/Cas9}

Currently, genome editing is widely used in studies involving functional genomics, transgenic animals, and gene therapy. Genome editing is based on programmable and highly specific nucleases, which generate site-specific cleavage and subsequently induce cellular DNA repair (Zhang et al., 2014). Multiple artificial nuclease systems have been developed for genome editing, including zinc-finger nucleases, tran- scription activator-like effector nucleases, and clustered regularly interspaced short palindromic repeats (CRISPR) and the CRISPR-associated (Cas) protein 9. Zinc-finger nucleases and transcription activator-like effector nucleases, based on protein-DNA interactions, are more complex and time-consuming compared with CRISPR/Cas9, which is easier and more efficient when using guide RNA (gRNA) and DNA targeting.

CRISPR/Cas9 is widely used in genetic modification, transcription regulation, and gene therapy studies. Researches have demonstrated that CRISPR/ Cas 9 can be used to conduct genomic editing in many organisms, including in bacteria (Jiang et al., 2013), drosophila (Gratz et al., 2013), zebrafish (Hruscha et al., 2013), mice (Wang H. et al., 2013), Caenorhabditis elegans (Friedland et al., 2013), and Bombyx mori (Wang Y. et al., 2013). Furthermore, in terms of the development of stem cell therapy, CRISPR/Cas9 has been widely applied in the accurate and complex genetic manipulation of stem cells to enhance their reprogramming, differentiation, and other functions. Mandal et al. (2014) successfully silenced the expression of the genes $B 2 M$ and $C C R 5$ in human hematopoietic cells using CRISPR/Cas9 with minimal off-target mutagenesis. Additionally, Wettstein et al. (2016) transfected two paired CRISPR single guide RNAs (sgRNAs)-Cas9 plasmids into mouse embryonic stem cells, which resulted in the knock-out of the targeted gene.

CRISPR/Cas9 provides us with a more efficient way to optimize MSC therapy for liver fibrosis. We can transform MSCs using different aspects to enhance their vitality and function, including their proliferation and differentiation ability, chemotaxis for injured tissue, and anti-inflammatory capacity. To aid in this, Schmidt et al. (2015) successfully built an arrayed sgRNA library that can target one critical exon of almost every protein-coding gene in humans. Therefore, by using the sgRNA library, we can find genes related to the various characteristics of MSCs, and then knockout the specific gene to optimize the MSC function.

It is also possible to take advantage of homologous recombination to overexpress targeted genes through CRISPR/Cas9. As mentioned above, genetically engineered MSCs that overexpress certain genes such as the genes for HGF and IGF-1 have therapeutic effects on liver fibrosis. However, it is 
unclear how we can overexpress specific genes stably without affecting the MSC function or the expression of other genes. This problem is critical. Currently, the use of recombinant virus infection is fervent, including the use of non-integrating viruses like RNA viruses, modified lentiviruses, and integrating adenoviruses (Seah et al., 2015). The efficiency of virus infection and the level of gene expression are both high; however, there are still some problems with this method. Non-integrating viruses will not integrate into the cell genome; therefore, the heterologous gene will not be stably expressed as cell proliferation. Thus, integrated adenoviruses are a good vector for targeted gene overexpression. However, adenoviruses, lentiviruses, and RNA viruses are all viruses, meaning that they are associated with pathogenic risks in clinical treatments. Therefore, finding a new method is necessary.

CRISPR/Cas9 is a promising tool that may allow us to transform MSCs in order to overexpress targeted genes. Currently, our lab is performing some related experiments. We have constructed a donor vector that contains the targeted gene, and next we will transfect it with the CRISPR sgRNAs-Cas9 plasmid into MSCs.
Taking advantage of homology-direct repair, targeted genes can be combined into the genomic DNA of the MSCs and stably expressed through proliferation (Fig. 3). Our goal is to obtain the targeted gene in a stably expressed cell line, which can then be used to treat liver fibrosis. However, the transfection efficiency is not high; hence, additional research is needed to improve the efficiency.

In general, CRISPR/Cas9 can be used to reform stem cells. Additionally, stem cell therapy combined with genomic editing will be a promising method for many diseases in the future.

\section{Current problems and future prospects}

The transplantation of MSCs for the treatment of liver fibrosis is an effective and promising method, considering the targeted migration ability, release capacity, and low immunogenicity of MSCs. MSCs can directly interact with the fibrogenic liver by differentiating into hepatocyte-like cells or by fusing with hepatocytes. Additionally, MSCs have the potential to release different growth factors and

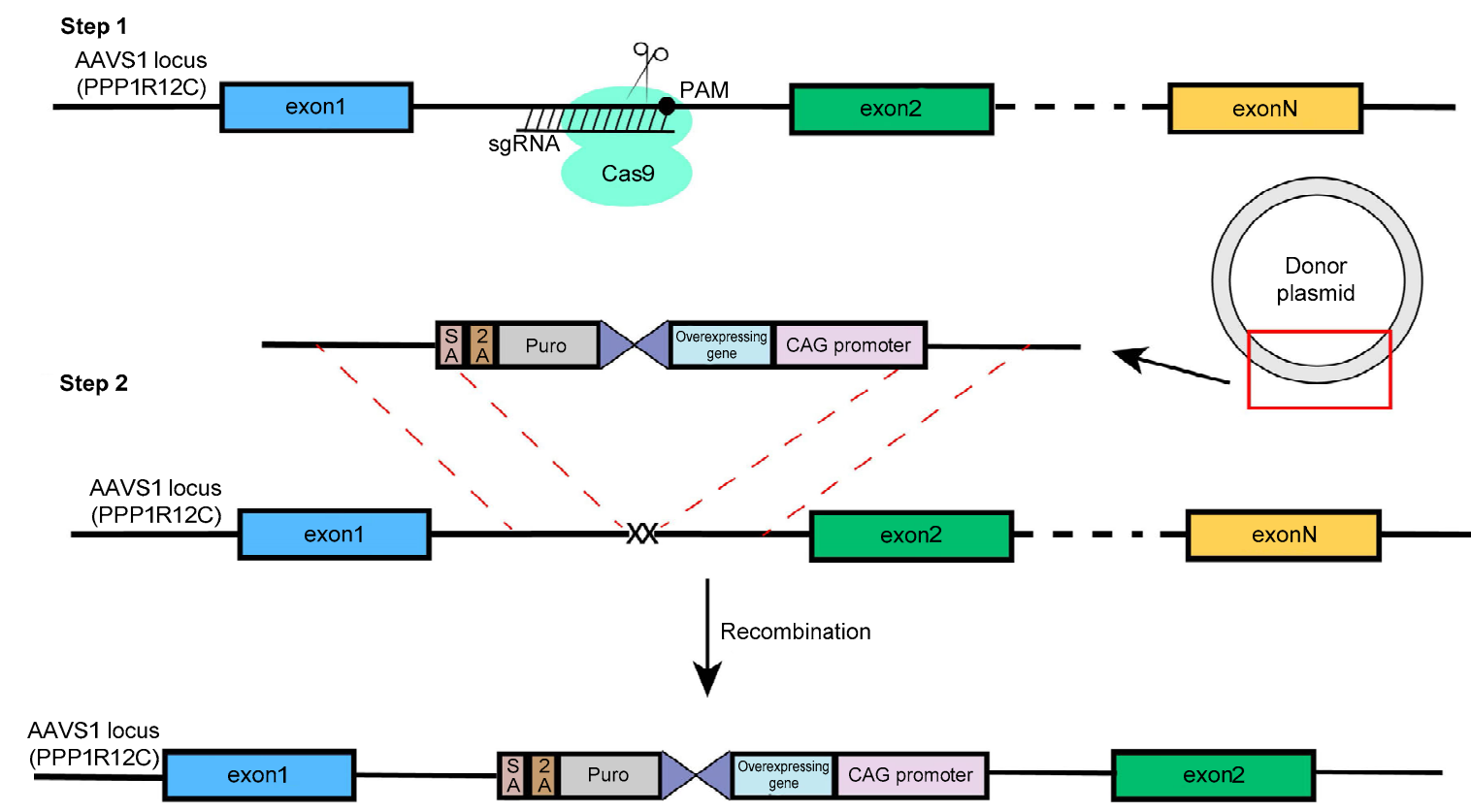

Fig. 3 Overexpressing gene in targeted site of genome through CRISPR/Cas9

The cleavage induced by CRISPR/Cas9 produces a double strand break, which will trigger cellular DNA repair processes, including non-homologous end-joining and homology-directed repairs. The AAVS1 locus is a safe harbor for insertion, and does not interfere with the expression of the inserted gene or other genes. We constructed a plasmid containing homologous arms of AAVS1 and inserted genes. Taking advantage of homology directed repair, we can insert certain genes into the specific site and obtain a stably expressed cell line 
cytokines, which can regulate the microenvironment and immune system to enhance their therapeutic effects on liver fibrosis. MSCs can also be combined with gene engineering to create a new method that can obviously regress fibrogenesis, promote regeneration, and restore the liver function. Therefore, MSC therapy for liver fibrosis is an optimal choice. However, many issues with these methods still need to be resolved. For instance, several different types of MSCs exist, which each have their respective advantages and disadvantages. The isolation of BM-MSCs is strenuous and traumatic. In contrast, MSCs derived from adipose tissue-derived MSCs are abundant and easily obtained, but the therapeutic effect is inferior to that of BM-MSCs (Liu et al., 2015). Moreover, we still do not fully understand the mechanisms underlying the therapeutic effects of MSCs. Therefore, the oncogenic potential and the risks of using MSCs remain unknown. In addition, when combining MSCs with gene engineering, the transfection problem exists, which will require finding a better transfection condition to increase the efficiency. In general, there is still much for us to explore regarding the use of MSCs in the treatment of liver fibrosis.

\section{Acknowledgements}

We thank Dr. Qiu-rong DING (Chinese Academy of Sciences in Shanghai, China) for his guidance on genome editing techniques. Additionally, we thank Editage (https://www. editage.com) for assistance with the English language editing.

\section{Compliance with ethics guidelines}

Yang GUO, Bo CHEN, Li-jun CHEN, Chun-feng ZHANG, and Charlie XIANG declare that they have no conflict of interest.

This article does not contain any studies with human or animal subjects performed by any of the authors.

\section{References}

Aggarwal, S., Pittenger, M.F., 2005. Human mesenchymal stem cells modulate allogeneic immune cell responses. Blood, 105(4): 1815-1822. http://dx.doi.org/10.1182/blood-2004-04-1559

Arthur, M.J., 1995. Collagenases and liver fibrosis. J. Hepatol., 22(2 Suppl.):43-48.

Arthur, M.J., 1997. Matrix degradation in liver: a role in injury and repair. Hepatology, 26(4):1069-1071. http://dx.doi.org/10.1053/jhep.1997.v26.ajhep0261069

Banas, A., Teratani, T., Yamamoto, Y., et al., 2007. Adipose tissue-derived mesenchymal stem cells as a source of human hepatocytes. Hepatology, 46(1):219-228. http://dx.doi.org/10.1002/hep.21704

Benyon, R.C., Iredale, J.P., Goddard, S., et al., 1996. Expression of tissue inhibitor of metalloproteinases 1 and 2 is increased in fibrotic human liver. Gastroenterology, 110(3):821-831.

Berardis, S., Dwisthi Sattwika, P., Najimi, M., et al., 2015. Use of mesenchymal stem cells to treat liver fibrosis: current situation and future prospects. World J. Gastroenterol., 21(3):742-758.

http://dx.doi.org/10.3748/wjg.v21.i3.742

Böker, K.H., Pehle, B., Steinmetz, C., et al., 2000. Tissue inhibitors of metalloproteinases in liver and serum/plasma in chronic active hepatitis $\mathrm{C}$ and $\mathrm{HCV}$-induced cirrhosis. Hepatogastroenterology, 47(33):812-819.

Bonefeld, K., Møller, S., 2011. Insulin-like growth factor-I and the liver. Liver Int., 31(7):911-919. http://dx.doi.org/10.1111/j.1478-3231.2010.02428.x

Campagnoli, C., Roberts, I.A., Kumar, S., et al., 2001. Identification of mesenchymal stem/progenitor cells in human first-trimester fetal blood, liver, and bone marrow. Blood, 98(8):2396-2402. http://dx.doi.org/10.1182/blood.V98.8.2396

Campard, D., Lysy, P.A., Najimi, M., et al., 2008. Native umbilical cord matrix stem cells express hepatic markers and differentiate into hepatocyte-like cells. Gastroenterology, 134(3):833-848. http://dx.doi.org/10.1053/j.gastro.2007.12.024

Chan, T.M., Harn, H.J., Lin, H.P., et al., 2014. Improved human mesenchymal stem cell isolation. Cell Transplant., 23(4-5):399-406. http://dx.doi.org/10.3727/096368914X678292

Corcione, A., Benvenuto, F., Ferretti, E., et al., 2006. Human mesenchymal stem cells modulate B-cell functions. Blood, 107(1):367-372. http://dx.doi.org/10.1182/blood-2005-07-2657

de Ugarte, D.A., Morizono, K., Elbarbary, A., et al., 2003. Comparison of multi-lineage cells from human adipose tissue and bone marrow. Cells Tissues Organs, 174(3): 101-109. http://dx.doi.org/10.1159/000071150

di Nicola, M., Carlo-Stella, C., Magni, M., et al., 2002. Human bone marrow stromal cells suppress T-lymphocyte proliferation induced by cellular or nonspecific mitogenic stimuli. Blood, 99(10):3838-3843. http://dx.doi.org/10.1182/blood.V99.10.3838

Ek, M., Soderdahl, T., Küppers-Munther, B., et al., 2007. Expression of drug metabolizing enzymes in hepatocyte-like cells derived from human embryonic stem cells. Biochem. Pharmacol., 74(3):496-503. http://dx.doi.org/10.1016/j.bcp.2007.05.009

Eom, Y.W., Kim, G., Baik, S.K., 2015. Mesenchymal stem cell therapy for cirrhosis: present and future perspectives. World J. Gastroenterol., 21(36):10253-10261. http://dx.doi.org/10.3748/wjg.v21.i36.10253

Erices, A., Conget, P., Minguell, J.J., 2000. Mesenchymal progenitor cells in human umbilical cord blood. Br. J. 
Haematol., 109(1):235-242.

http://dx.doi.org/10.1046/j.1365-2141.2000.01986.x

Fiore, E.J., Bayo, J.M., Garcia, M.G., et al., 2015. Mesenchymal stromal cells engineered to produce IGF-I by recombinant adenovirus ameliorate liver fibrosis in mice. Stem Cells Dev., 24(6):791-801. http://dx.doi.org/10.1089/scd.2014.0174

Forbes, S.J., Russo, F.P., Rey, V., et al., 2004. A significant proportion of myofibroblasts are of bone marrow origin in human liver fibrosis. Gastroenterology, 126(4):955-963. http://dx.doi.org/10.1053/j.gastro.2004.02.025

Friedland, A.E., Tzur, Y.B., Esvelt, K.M., et al., 2013. Heritable genome editing in C. elegans via a CRISPRCas9 system. Nat. Methods, 10(8):741-743. http://dx.doi.org/10.1038/nmeth.2532

Friedman, S.L., 1993. Seminars in medicine of the Beth Israel Hospital, Boston. The cellular basis of hepatic fibrosis. Mechanisms and treatment strategies. N. Engl. J. Med., 328(25): $1828-1835$. http://dx.doi.org/10.1056/NEJM199306243282508

Glennie, S., Soeiro, I., Dyson, P.J., et al., 2005. Bone marrow mesenchymal stem cells induce division arrest anergy of activated T cells. Blood, 105(7):2821-2827. http://dx.doi.org/10.1182/blood-2004-09-3696

Golzar, F., Javanmard, S.H., Bahrambeigi, V., et al., 2015. The effect of Kisspeptin-10 on mesenchymal stem cells migration in vitro and in vivo. Adv. Biomed. Res., 4:20. http://dx.doi.org/10.4103/2277-9175.149851

Gratz, S.J., Cummings, A.M., Nguyen, J.N., et al., 2013. Genome engineering of Drosophila with the CRISPR RNA-guided Cas9 nuclease. Genetics, 194(4):1029-1035. http://dx.doi.org/10.1534/genetics.113.152710

Groh, M.E., Maitra, B., Szekely, E., et al., 2005. Human mesenchymal stem cells require monocyte-mediated activation to suppress alloreactive T cells. Exp. Hematol., 33(8):928-934. http://dx.doi.org/10.1016/j.exphem.2005.05.002

Hang, H., Yu, Y., Wu, N., et al., 2014. Induction of highly functional hepatocytes from human umbilical cord mesenchymal stem cells by HNF $4 \alpha$ transduction. PLOS ONE, 9(8):e104133. http://dx.doi.org/10.1371/journal.pone.0104133

Hay, E.D., 2005. The mesenchymal cell, its role in the embryo, and the remarkable signaling mechanisms that create it. Dev. Dyn., 233(3):706-720. http://dx.doi.org/10.1371/10.1002/dvdy.20345

Hengstler, J.G., Brulport, M., Schormann, W., et al., 2005. Generation of human hepatocytes by stem cell technology: definition of the hepatocyte. Expert Opin. Drug Metab. Toxicol., 1(1):61-74. http://dx.doi.org/10.1517/17425255.1.1.61

Hruscha, A., Krawitz, P., Rechenberg, A., et al., 2013. Efficient CRISPR/Cas9 genome editing with low off-target effects in zebrafish. Development, 140(24):4982-4987. http://dx.doi.org/10.1242/dev.099085

Huang, X., Liu, L.I., Liu, N., 2015. Molecular mechanism of tumor necrosis factor-alpha monoclonal antibody in hepatopulmonary syndrome in rats. Chin. J. Hepatol., 23(6):458-463 (in Chinese).

Iredale, J.P., Murphy, G., Hembry, R.M., et al., 1992. Human hepatic lipocytes synthesize tissue inhibitor of metalloproteinases-1. Implications for regulation of matrix degradation in liver. J. Clin. Invest., 90(1):282-287. http://dx.doi.org/10.1172/JCI115850

Ishii, K., Yoshida, Y., Akechi, Y., et al., 2008. Hepatic differentiation of human bone marrow-derived mesenchymal stem cells by tetracycline-regulated hepatocyte nuclear factor 3ß. Hepatology, 48(2):597-606. http://dx.doi.org/10.1002/hep.22362

Jang, Y.O., Kim, M.Y., Cho, M.Y., et al., 2014. Effect of bone marrow-derived mesenchymal stem cells on hepatic fibrosis in a thioacetamide-induced cirrhotic rat model. BMC Gastroenterol., 14(1):198. http://dx.doi.org/10.1186/s12876-014-0198-6

Jiang, W.Y., Bikard, D., Cox, D., et al., 2013. RNA-guided editing of bacterial genomes using CRISPR-Cas systems. Nat. Biotechnol., 31(3):233-239. http://dx.doi.org/10.1038/nbt.2508

Jiang, X.X., Zhang, Y., Liu, B., et al., 2005. Human mesenchymal stem cells inhibit differentiation and function of monocyte-derived dendritic cells. Blood, 105(10):4120-4126. http://dx.doi.org/10.1182/blood-2004-02-0586

Jiang, Y.H., Vaessen, B., Lenvik, T., et al., 2002. Multipotent progenitor cells can be isolated from postnatal murine bone marrow, muscle, and brain. Exp. Hematol., 30(8): 896-904.

Kakinuma, S., Nakauchi, H., Watanabe, M., 2009. Hepatic stem/progenitor cells and stem-cell transplantation for the treatment of liver disease. J. Gastroenterol., 44(3):167-172. http://dx.doi.org/10.1007/s00535-008-2297-z

Kelly, J.D., Haldeman, B.A., Grant, F.J., et al., 1991. Plateletderived growth factor (PDGF) stimulates PDGF receptor subunit dimerization and intersubunit trans-phosphorylation. J. Biol. Chem., 266(14):8987-8992.

Kharaziha, P., Hellstrom, P.M., Noorinayer, B., et al., 2009. Improvement of liver function in liver cirrhosis patients after autologous mesenchymal stem cell injection: a phase I-II clinical trial. Eur. J. Gastroenterol. Hepatol., 21(10):1199-1205. http://dx.doi.org/10.1097/MEG.0b013e32832a1f6c

Kim, M.D., Kim, S.S., Cha, H.Y., et al., 2014. Therapeutic effect of hepatocyte growth factor-secreting mesenchymal stem cells in a rat model of liver fibrosis. Exp. Mol. Med., 46:e110. http://dx.doi.org/10.1038/emm.2014.49

Kiss, J., Urbán, V.S., Dudics, V., et al., 2008. Mesenchymal stem cells and the immune system-immunosuppression without drugs? Orv. Hetil., 149(8):339-346 (in Hungarian). http://dx.doi.org/10.1556/OH.2008.28291

Krampera, M., Glennie, S., Dyson, J., et al., 2003. Bone marrow mesenchymal stem cells inhibit the response of 
naive and memory antigen-specific $\mathrm{T}$ cells to their cognate peptide. Blood, 101(9):3722-3729. http://dx.doi.org/10.1182/blood-2002-07-2104

Krampera, M., Cosmi, L., Angeli, R., et al., 2006. Role for interferon- $\gamma$ in the immunomodulatory activity of human bone marrow mesenchymal stem cells. Stem Cells, 24(2):386-398. http://dx.doi.org/10.1634/stemcells.2005-0008

Lian, G.W., Wang, C.Y., Teng, C.B., et al., 2006. Failure of hepatocyte marker-expressing hematopoietic progenitor cells to efficiently convert into hepatocytes in vitro. Exp. Hematol., 34(3):348-358. http://dx.doi.org/10.1016/j.exphem.2005.12.004

Lichtinghagen, R., Michels, D., Haberkorn, C.I., et al., 2001. Matrix metalloproteinase (MMP)-2, MMP-7, and tissue inhibitor of metalloproteinase- 1 are closely related to the fibroproliferative process in the liver during chronic hepatitis C. J. Hepatol., 34(2):239-247. http://dx.doi.org/10.1016/S0168-8278(00)00037-4

Lin, H., Xu, R., Zhang, Z., et al., 2011. Implications of the immunoregulatory functions of mesenchymal stem cells in the treatment of human liver diseases. Cell. Mol. Immunol., 8(1):19-22. http://dx.doi.org/10.1038/cmi.2010.57

Liu, W.H., Song, F.Q., Ren, L.N., et al., 2015. The multiple functional roles of mesenchymal stem cells in participating in treating liver diseases. J. Cell. Mol. Med., 19(3): 511-520. http://dx.doi.org/10.1111/jcmm.12482

Liu, Y., Zuo, G.Q., Zhao, L., et al., 2013. Effect of inflammatory stress on hepatic cholesterol accumulation and hepatic fibrosis in C57BL/6J mice. Chin. J. Hepatol., 21(2):116-120 (in Chinese). http://dx.doi.org/10.3760/cma.j.issn.1007-3418.2013.02.010

Lourenco, S., Teixeira, V.H., Kalber, T., et al., 2015. Macrophage migration inhibitory factor-CXCR4 is the dominant chemotactic axis in human mesenchymal stem cell recruitment to tumors. J. Immunol., 194(7):3463-3474. http://dx.doi.org/10.4049/jimmunol.1402097

Mandal, P.K., Ferreira, L.M., Collins, R., et al., 2014. Efficient ablation of genes in human hematopoietic stem and effector cells using CRISPR/Cas9. Cell Stem Cell, 15(5): 643-652. http://dx.doi.org/10.1016/j.stem.2014.10.004

Meisel, R., Zibert, A., Laryea, M., et al., 2004. Human bone marrow stromal cells inhibit allogeneic T-cell responses by indoleamine 2,3-dioxygenase-mediated tryptophan degradation. Blood, 103(12):4619-4621. http://dx.doi.org/10.1182/blood-2003-11-3909

Milani, S., Herbst, H., Schuppan, D., et al., 1994. Differential expression of matrix-metalloproteinase- 1 and -2 genes in normal and fibrotic human liver. Am. J. Pathol., 144(3): 528-537.

Mohsin, S., Shams, S., Ali Nasir, G., et al., 2011. Enhanced hepatic differentiation of mesenchymal stem cells after pretreatment with injured liver tissue. Differentiation,
81(1):42-48.

http://dx.doi.org/10.1016/j.diff.2010.08.005

Mou, X.Z., Lin, J., Chen, J.Y., et al., 2013. Menstrual blood-derived mesenchymal stem cells differentiate into functional hepatocyte-like cells. J. Zhejiang Univ.-Sci. B (Biomed. \& Biotechnol.), 14(11):961-972. http://dx.doi.org/10.1631/jzus.B1300081

Nauta, A.J., Kruisselbrink, A.B., Lurvink, E., et al., 2006. Mesenchymal stem cells inhibit generation and function of both $\mathrm{CD} 34^{+}$-derived and monocyte-derived dendritic cells. J. Immunol., 177(4):2080-2087. http://dx.doi.org/10.4049/jimmunol.177.4.2080

Ortiz, L.A., DuTreil, M., Fattman, C., et al., 2007. Interleukin 1 receptor antagonist mediates the antiinflammatory and antifibrotic effect of mesenchymal stem cells during lung injury. PNAS, 104(26):11002-11007. http://dx.doi.org/10.1073/pnas.0704421104

Parekkadan, B., van Poll, D., Megeed, Z., et al., 2007. Immunomodulation of activated hepatic stellate cells by mesenchymal stem cells. Biochem. Biophys. Res. Commun., 363(2):247-252. http://dx.doi.org/10.1016/j.bbrc.2007.05.150

Potian, J.A., Aviv, H., Ponzio, N.M., et al., 2003. Veto-like activity of mesenchymal stem cells: functional discrimination between cellular responses to alloantigens and recall antigens. J. Immunol., 171(7):3426-3434.

Puglisi, M.A., Tesori, V., Lattanzi, W., et al., 2011. Therapeutic implications of mesenchymal stem cells in liver injury. J. Biomed. Biotechnol., 2011:860578. http://dx.doi.org/10.1155/2011/860578

Rasmusson, I., Ringdén, O., Sundberg, B., et al., 2003. Mesenchymal stem cells inhibit the formation of cytotoxic $\mathrm{T}$ lymphocytes, but not activated cytotoxic $\mathrm{T}$ lymphocytes or natural killer cells. Transplantation, 76(8):1208-1213. http://dx.doi.org/10.1097/01.TP.0000082540.43730.80

Reinders, M.E., Rabelink, T.J., de Fijter, J.W., 2013. The role of mesenchymal stromal cells in chronic transplant rejection after solid organ transplantation. Curr. Opin. Organ Transplant., 18(1):44-50. http://dx.doi.org/10.1097/MOT.0b013e32835c2939

Schmidt, T., Schmid-Burgk, J.L., Hornung, V., 2015. Synthesis of an arrayed sgRNA library targeting the human genome. Sci. Rep., 5:14987. http://dx.doi.org/10.1038/srep14987

Seah, Y.F.S., el Farran, C.A., Warrier, T., et al., 2015. Induced pluripotency and gene editing in disease modelling: perspectives and challenges. Int. J. Mol. Sci., 16(12): 28614-28634. http://dx.doi.org/10.3390/ijms161226119

Sharma, R.R., Pollock, K., Hubel, A., et al., 2014. Mesenchymal stem or stromal cells: a review of clinical applications and manufacturing practices. Transfusion, 54(5):1418-1437. http://dx.doi.org/10.1111/trf.12421

Shi, M., Liu, Z.W., Wang, F.S., 2011. Immunomodulatory properties and therapeutic application of mesenchymal stem cells. Clin. Exp. Immunol., 164(1):1-8. 
http://dx.doi.org/10.1111/j.1365-2249.2011.04327.x

Siller-López, F., Sandoval, A., Salgado, S., et al., 2004. Treatment with human metalloproteinase- 8 gene delivery ameliorates experimental rat liver cirrhosis. Gastroenterology, 126(4):1122-1133; discussion 1949. http://dx.doi.org/10.1053/j.gastro.2003.12.045

Snykers, S., Vanhaecke, T., de Becker, A., et al., 2007. Chromatin remodeling agent trichostatin A: a key-factor in the hepatic differentiation of human mesenchymal stem cells derived of adult bone marrow. BMC Dev. Biol., 7:24.

http://dx.doi.org/10.1186/1471-213X-7-24

Sotiropoulou, P.A., Perez, S.A., Gritzapis, A.D., et al., 2006. Interactions between human mesenchymal stem cells and natural killer cells. Stem Cells, 24(1):74-85. http://dx.doi.org/10.1634/stemcells.2004-0359

Tse, W.T., Pendleton, J.D., Beyer, W.M., et al., 2003. Suppression of allogeneic T-cell proliferation by human marrow stromal cells: implications in transplantation. Transplantation, 75(3):389-397. http://dx.doi.org/10.1097/01.TP.0000045055.63901.A9

Wang, H., Yang, H., Shivalila, C.S., et al., 2013. One-step generation of mice carrying mutations in multiple genes by CRISPR/Cas-mediated genome engineering. Cell, 153(4): 910-918. http://dx.doi.org/10.1016/j.cell.2013.04.025

Wang, Y., Li, Z., Xu, J., et al., 2013. The CRISPR/Cas system mediates efficient genome engineering in Bombyx mori. Cell Res., 23(12):1414-1416. http://dx.doi.org/10.1038/cr.2013.146

Wells, R.G., Kruglov, E., Dranoff, J.A., 2004. Autocrine release of TGF- $\beta$ by portal fibroblasts regulates cell growth. FEBS Lett., 559(1-3):107-110. http://dx.doi.org/10.1016/S0014-5793(04)00037-7

Wettstein, R., Bodak, M., Ciaudo, C., 2016. Generation of a knockout mouse embryonic stem cell line using a paired CRISPR/Cas9 genome engineering tool. Methods Mol. Biol., 1341:321-343. http://dx.doi.org/10.1007/7651_2015_213

Wrana, J.L., 1999. Transforming growth factor- $\beta$ signaling and cirrhosis. Hepatology, 29(6):1909-1910. http://dx.doi.org/10.1002/hep.510290641

Yin, L.B., Zhu, Y.H., Yang, J.G., et al., 2015. Adipose tissue-derived mesenchymal stem cells differentiated into hepatocyte-like cells in vivo and in vitro. Mol. Med. Rep.,
11(3):1722-1732.

http://dx.doi.org/10.3892/mmr.2014.2935

Yoshida, Y., Shimomura, T., Sakabe, T., et al., 2007. A role of $\mathrm{Wnt} / \beta$-catenin signals in hepatic fate specification of human umbilical cord blood-derived mesenchymal stem cells. Am. J. Physiol. Gastrointest. Liver Physiol., 293(5): G1089-G1098. http://dx.doi.org/10.1152/ajpgi.00187.2007

Zappia, E., Casazza, S., Pedemonte, E., et al., 2005. Mesenchymal stem cells ameliorate experimental autoimmune encephalomyelitis inducing T-cell anergy. Blood, 106(5): 1755-1761.

http://dx.doi.org/10.1182/blood-2005-04-1496

Zhang, F., Wen, Y., Guo, X., 2014. CRISPR/Cas9 for genome editing: progress, implications and challenges. Hum. Mol. Genet., 23(R1):R40-R46.

http://dx.doi.org/10.1093/hmg/ddu125

Zhang, W., Ge, W., Li, C.H., et al., 2004. Effects of mesenchymal stem cells on differentiation, maturation, and function of human monocyte-derived dendritic cells. Stem Cells Dev., 13(3):263-271. http://dx.doi.org/10.1089/154732804323099190

Zhou, P., Hohm, S., Olusanya, Y., et al., 2009. Human progenitor cells with high aldehyde dehydrogenase activity efficiently engraft into damaged liver in a novel model. Hepatology, 49(6):1992-2000. http://dx.doi.org/10.1002/hep.22862

\section{中文概要}

题 目: 间充质干细胞治疗肝纤维化的现况和前景

概 要: 许多慢性肝病发展到终末阶段形成肝纤维化进而 转变成肝硬化, 严重威胁人们的健康。目前临床 治疗肝纤维化的有效方法是肝移植, 但由于供体 缺乏、免疫排斥和治疗费用昂贵等诸多缺陷, 这 种疗法并不是一种理想的治疗途径。近些年细胞 治疗研究火热, 间充质干细胞作为一种来源广 泛、可定向迁移和多向分化的治疗载体, 在肝纤 维化治疗上具有广阔的应用前景。本文对间充质 干细胞治疗肝纤维化的现状进行归纳, 并对其可 行性、局限性及应用前景进行分析。

关键词: 间充质干细胞; 肝纤维化; 细胞治疗 